\title{
La suplementación de aceite esencial de orégano en la dieta reduce el estrés oxidativo en la yema de huevo y mejora los parámetros productivos de la codorniz japonesa (Coturnix coturnix japonica)
}

\author{
Supplementation of oregano essential oil in the diet reduces oxidative stress \\ in the egg yolk and improves the productive performance of the \\ Japanese quail (Coturnix coturnix japonica)
}

\author{
Bruno Loyaga-Cortéz ${ }^{1,3}$, Gilmar Mendoza Ordoñez, Roberto Ybañez-Julca ${ }^{2}$, \\ Daniel Asunción-Alvarez ${ }^{2}$
}

\section{Resumen}

\begin{abstract}
El objetivo de este estudio fue evaluar el efecto de la suplementación con aceite esencial de orégano en la dieta de codornices sobre el rendimiento y la estabilidad oxidativa del huevo. Se utilizaron 300 codornices de 110 días de edad, distribuidas en cinco grupos: Control (dieta sin suplementación), E\&C (dieta más $0.01 \%$ de enramicina y $0.025 \%$ de sulfato de colistina) y tratamientos A, B y C (dietas suplementadas con 100, 150 y $200 \mathrm{mg} / \mathrm{kg}$ de aceite esencial de orégano, respectivamente). La administración de los tratamientos se realizó durante 12 semanas. La estabilidad oxidativa de la yema de huevo se evaluó en los días 0,7 y 15 de almacenamiento. Los resultados mostraron que la suplementación dietética con aceite esencial de orégano mejoró los parámetros productivos en comparación al control ( $\mathrm{p}<0.05)$, aunque fue superado por E\&C. El grupo A $(100 \mathrm{mg} / \mathrm{kg})$ disminuyó la peroxidación lipídica en la yema de huevo en los días $0(p<0.0001), 7(p<0.01)$ y 15 $(\mathrm{p}<0.0001)$, respecto al control e incluso fue mejor que $\mathrm{E} \& \mathrm{C}(\mathrm{p}<0.05)$; sin embargo, este efecto no presentó relación directa con el tiempo de almacenamiento. En conclusión, el aceite esencial de orégano puede ser un suplemento nutricional potencial y también un antioxidante que serviría para reducir la oxidación de los lípidos en la yema de huevo y mejorar ciertos parámetros de producción.
\end{abstract}

Palabras clave: aceite esencial de orégano, antioxidantes naturales, oxidación de lípidos, parámetros productivos de codorniz

\footnotetext{
${ }^{1}$ Departamento de Agronomía y Zootecnia, Facultad de Ciencias Agropecuarias, Universidad Nacional de Trujillo, La Libertad, Trujillo, Perú

${ }^{2}$ Departamento de Farmacología, Facultad de Farmacia y Bioquímica, Universidad Nacional de Trujillo, La Libertad, Trujillo, Perú

${ }^{3}$ E-mail: bruno_loyaga@outlook.com
}

Recibido: 1 de septiembre de 2019

Aceptado para publicación: 22 de mayo de 2020

Publicado: 11 de agosto de 2020 
The aim of this study was to evaluate the effect of supplementation with oregano essential oil in the quail diet on the performance and oxidative stability of the egg. A total of 300 quail, 110 days old, were distributed in five groups: Control (diet without supplementation), E\&C (diet plus $0.01 \%$ enramycin and $0.025 \%$ colistin sulfate) and treatments A, B and C (diets supplemented with 100, 150 and $200 \mathrm{mg} / \mathrm{kg}$ of oregano essential oil, respectively). The study was carried out for 12 weeks. The oxidative stability of the egg yolk was evaluated on days 0,7 and 15 of storage. The results showed that dietary supplementation with essential oil of oregano improved productive parameters compared to control $(\mathrm{p}<0.05)$, although it was surpassed by E\&C. Group A $(100 \mathrm{mg} / \mathrm{kg})$ decreased lipid peroxidation in egg yolk on days $0(p<0.0001), 7(p<0.01)$ and $15(p<0.0001)$, with respect to the control and was even better than $\mathrm{E} \& \mathrm{C}(\mathrm{p}<0.05)$; however, this effect was not directly related to storage time. In conclusion, oregano essential oil can be a potential nutritional supplement and an antioxidant that would help reduce lipid oxidation in egg yolk and improve certain production parameters.

Key words: oregano essential oil, natural antioxidants, lipid oxidation, quail productive performance

\section{INTRODUCCIÓN}

Origanum vulgare L, comúnmente conocido como «orégano», es una especie herbácea mediterránea de la familia Lamiaceae (Oniga et al., 2018), que se utiliza en medicina tradicional para diversos tipos de trastornos (Marwat et al., 2009; Lukas et al., 2015). Existe evidencia que el orégano posee actividad antioxidante, antimicrobiana y hepatoprotectora (Oniga et al., 2018), además de efectos tales como antiinflamatorio, cardioprotector, antimutagénico, antiproliferativo, inhibidor de la $\alpha$-amilasa y $\alpha$ glucosidasa (Leyva-López et al., 2017), antiviral (Zhang et al., 2014), antiespasmódico (Gonceariuc et al., 2015), antiurolítico (Khan et al., 2011) y neuroprotector (Gîrd et al., 2016), entre otros. La composición del aceite esencial de orégano lo constituyen principalmente terpenos, generalmente mono y sesquiterpenos (Marwat et al., 2009).
Por otro lado, en la nutrición de las aves se requiere incluir ingredientes que maximicen la disponibilidad de nutrientes (Beski et al., 2015). Esto se puede lograr con la adición de componentes fitogenéticos con propiedades antioxidantes y que mejoren la inmunidad humoral (Saleh et al., 2018), e incluso que puedan servir como promotores de crecimiento (Dibner y Richards, 2005). Actualmente se reconoce que el uso masivo de antibióticos ha llevado a un mayor problema de resistencia, y la presencia de residuos de antibióticos en los alimentos y el medio ambiente, compromete la salud humana y animal (Mehdi et al., 2018). Debido al riesgo que representan los antibióticos en la dieta de los animales y con base al 'principio de precaución', la Comisión Europea prohibió desde el 1 de enero de 2006 la comercialización y el uso de antimicrobianos como promotores de crecimiento (Anadón, 2006), facilitando la investigación en productos alternativos (Millet y Maertens, 2011). 
Los productos alternativos no solo se enfocan en la mejora del crecimiento de las aves sino también de sus productos, como es el caso de los huevos de la codorniz (Coturnix coturnix japonica), los cuales tienen menor contenido de grasa en comparación con los huevos de gallina (Tolik et al., 2014). Se sabe que la oxidación de los lípidos es una de las principales causas del deterioro de los alimentos que contienen grasa, especialmente aquellos que contienen ácidos grasos poliinsaturados (PUFA) (Papastergiadis et al., 2012). Estas reacciones oxidativas producen una amplia variedad de productos primarios como los hidroperóxidos de lípidos ( $\mathrm{LOOH})$, pero también productos secundarios como el malondialdehído (MDA) y el 4-hidroxinonenal (Ayala et al., 2014). Diversos estudios refuerzan la idea de que el MDA sería capaz de producir mutaciones, sustitución de pares de bases y otros mecanismos de genotoxicidad, que están involucrados con su capacidad de «entrecruzarse» a nivel de ADN (Del Rio et al., 2005). Es por ello por lo que los componentes naturales como sustitutos de los aditivos sintéticos vienen adquiriendo mayor relevancia (Radwan et al., 2008).

En los últimos años, el aceite esencial de orégano ha sido uno de los principales suplementos dietéticos y del que existen suficientes evidencias sobre sus propiedades antimicrobianas y antioxidantes. A pesar de esto, hay muy pocos estudios que corroboren y relacionen este efecto en modelos experimentales de codornices. El objetivo del presente estudio fue utilizar el aceite esencial de orégano en la alimentación de codornices para promover el rendimiento y retrasar la oxidación de los lípidos en los huevos para dilucidar si los componentes antioxidantes presentes en el aceite esencial podrían reemplazar el uso total o parcial de sintéticos antioxidantes.

\section{Materiales y MéTodos}

Las codornices (Coturnix japonica) fueron proporcionadas por una granja del Valle de León Encantado (Trujillo, Perú). La evaluación de los parámetros productivos de las aves se llevó a cabo en el Laboratorio de Nutrición Animal de la Facultad de Ciencias Agropecuarias y la prueba de estabilidad oxidativa en el Laboratorio de Farmacología de la Facultad de Farmacia y Bioquímica de la Universidad Nacional de Trujillo, Perú. El estudio fue aprobado por un comité de ética animal y se adhirió al Código de Ética para la Investigación de la Universidad Nacional de Trujillo.

\section{Animales, Dietas y Diseño Experimental}

Trescientas codornices japonesas de 110 días de edad se distribuyeron en cinco grupos similares. Las aves se alojaron en jaulas $(150 \times 46 \times 35 \mathrm{~cm})$ en grupos de 60 , en condiciones convencionales y con acceso a alimento y agua ad libitum. Las dietas experimentales se ofrecieron durante 12 semanas, tiempo en que la luz diurna fue de 12 horas. Las dietas (Cuadro 1) se prepararon mensualmente, utilizando un mezclador de 200 $\mathrm{kg}$ de capacidad. Las dietas, tipo polvo, se formularon utilizando el software ZMix iT ${ }^{\circledR}$ v. 3.1 (Zootech, Lima, Perú) con el contenido nutricional indicado en el Cuadro 2.

El aceite esencial de orégano (Origanum vulgare) microencapsulado fue proveído por un laboratorio (Shandong Luxi Animal Medicine Share, Shandong, China). La dieta experimental se formuló de acuerdo con los requerimientos indicados por Rostagno et al . (2017). Los tratamientos fueron:

- Control: Solo la dieta.

- $\quad$ E\&C: Dieta + dos antibióticos $(0.01 \%$ de enramicina y $0.025 \%$ de sulfato de colistina). 
Cuadro 1. Composición de la dieta experimental ofrecida a codornices (Coturnix japonica)

\begin{tabular}{lc}
\hline Ingredientes & $\begin{array}{c}\text { Cantidad } \\
(\%)\end{array}$ \\
\hline Maíz & 42.00 \\
Afrecho & 12.60 \\
Torta de soya & 24.80 \\
Soya integral & 5.00 \\
Fosfato monocálcico & 0.45 \\
Carbonato de calcio & 4.50 \\
(polvo) & \\
Carbonato de calcio & 4.13 \\
(granulado) & 5.00 \\
Aceite de soya & 0.32 \\
DL-Metionina & 0.28 \\
L-Lisina & 0.25 \\
Sal & 0.10 \\
Vitaminas y minerales* & 0.20 \\
Cloruro de colina & 0.20 \\
Bicarbonato de sodio & 0.20 \\
Aluminosilicatos & 100.0 \\
\hline Total
\end{tabular}

* Vitaminas y mezcla de minerales: por kg contiene vitamina A 9000000 UI, vitamina D3 250000 UI, vitamina D $15000 \mathrm{UI}$, vitamina K3 $2.5 \mathrm{~g}$, vitamina B1 $1.5 \mathrm{~g}$, vitamina B2 $6.5 \mathrm{~g}$, vitamina B6 $2 \mathrm{~g}$, vitamina B12 $0.01 \mathrm{~g}$, Vitamina B5 $5.50 \mathrm{~g}$, Vitamina B9 $1 \mathrm{~g}$, Vitamina B3 $25 \mathrm{~g}$, Vitamina B8 $0.15 \mathrm{~g}$, Manganeso 70 g, Zinc 70 g, Hierro 30 g, Cobre $8 \mathrm{~g}$, Yodo $1 \mathrm{~g}$, Selenio $0,30 \mathrm{~g}$, Cobalto $0.1 \mathrm{~g}$, Excipientes

- A: Dieta con suplemento de $100 \mathrm{mg} / \mathrm{kg}$ de aceite esencial de orégano.

- B: Dieta con suplemento de $150 \mathrm{mg} / \mathrm{kg}$ de aceite esencial de orégano.
- C: Dieta con suplemento de $200 \mathrm{mg} / \mathrm{kg}$ de aceite esencial de orégano.

\section{Variables en Estudio}

La ingesta de alimentos (g) y la masa de huevos $(\mathrm{g})$ se registraron semanalmente. La conversión de alimento se calculó semanalmente como alimento de consumo de alimento (g) dividido por la producción de huevos $(\mathrm{g})$ de cada tratamiento. La producción de huevos (\%) y el peso de los huevos (g) se registraron diariamente. De cada grupo experimental, se tomaron muestras de 10 huevos al azar para la prueba de estabilidad oxidativa mediante el método TBARS (sustancias reactivas al ácido tiobarbitúrico).

\section{Sustancias Reactivas al Ácido Tiobar- bitúrico}

Las sustancias reactivas al ácido tiobarbitúrico (TBARS) como indicador de oxidación de lípidos se evaluaron utilizando la metodología descrita por Prabhakar et al. (2012) con algunas modificaciones. En resumen, el último día del periodo experimental se seleccionaron 10 huevos por cada nivel de aceite esencial de orégano. Estos huevos fueron analizados a los 0,7 y 15 días de almacenamiento a temperatura ambiente, en un lugar

Cuadro 2. Composición nutricional de la dieta experimental

\begin{tabular}{lc}
\hline Componente & $\begin{array}{c}\text { Cantidad } \\
(\%)\end{array}$ \\
\hline Proteína cruda & 18.92 \\
Energía (Mcal/kg dieta) & 2.82 \\
Materia seca & 89.71 \\
Fosforo disponible & 0.23 \\
Calcio & 3.12 \\
Lisina & 1.09 \\
Metionina & 0.56 \\
Metionina + Cisteína & 0.79 \\
\hline
\end{tabular}


Cuadro 3. Efecto del aceite esencial de Origanum vulgare en la dieta sobre los parámetros productivos de codornices (Coturnix japonica)

\begin{tabular}{lccccc}
\hline Tratamientos & EP & EW & EM & FC & FI \\
\hline Control & $81.54 \pm 0.23^{\mathrm{d}}$ & $10.81 \pm 0.12$ & $8.81 \pm 0.10^{\mathrm{b}}$ & $2.79 \pm 0.03^{\mathrm{a}}$ & $24.57 \pm 0.01^{\mathrm{b}}$ \\
E\&C & $92.09 \pm 0.75^{\mathrm{a}}$ & $11.13 \pm 0.14$ & $10.25 \pm 0.25^{\mathrm{a}}$ & $2.41 \pm 0.02^{\mathrm{c}}$ & $24.64 \pm 0.03^{\mathrm{b}}$ \\
A & $87.17 \pm 0.86^{\mathrm{c}}$ & $11.16 \pm 0.03$ & $9.73 \pm 0.23^{\mathrm{a}}$ & $2.54 \pm 0.03^{\mathrm{b}}$ & $24.67 \pm 0.04^{\mathrm{b}}$ \\
B & $89.22 \pm 0.72^{\mathrm{bc}}$ & $11.01 \pm 0.05$ & $9.82 \pm 0.18^{\mathrm{a}}$ & $2.51 \pm 0.02^{\mathrm{bc}}$ & $24.61 \pm 0.03^{\mathrm{b}}$ \\
C & $91.43 \pm 0.75^{\mathrm{ab}}$ & $11.08 \pm 0.11$ & $10.03 \pm 0.28^{\mathrm{a}}$ & $2.47 \pm 0.03^{\mathrm{bc}}$ & $24.92 \pm 0.10^{\mathrm{a}}$ \\
\hline P-value & $<0.0001$ & 0.1163 & 0.0002 & $<0.0001$ & 0.0002 \\
\hline
\end{tabular}

$\mathrm{EP}=$ Producción de huevos (\%); EW = Peso del huevo (g/día); EM = Masa de huevo (g/día/ave);

$\mathrm{FC}=$ Conversión alimenticia ( $\mathrm{kg}$ alimento/kg huevo); $\mathrm{FI}=$ Consumo de alimento (g/día)

Los valores son medias de 12 repeticiones $(n=60) \pm$ error estándar de la media

$a, b, c, d$ Los promedios con diferentes superíndices dentro de columnas difieren significativamente $(p<0.05)$

Control: Solo la dieta; E\&C: Dieta + dos antibióticos, A, B y C: Dieta con 100, 150 y 200 mg/kg de aceite esencial de orégano, respectivamente

fresco y seco con sombra, para determinar la concentración de malonaldehído por mililitro de yema. La yema de huevo (1.65 g) fue homogenizada con $5 \mathrm{ml}$ de agua desionizada y se colocó $0.5 \mathrm{ml}$ en un tubo de ensayo. Se agregó $1 \mathrm{ml}$ de solución de TBA-TCA (ácido tiobarbitúrico $20 \mathrm{mM}$ en ácido tricloroacético al $15 \%[\mathrm{p} / \mathrm{v}])$. Las muestras fueron colocadas en baño maría $\left(90^{\circ} \mathrm{C}\right.$ durante 15 minutos), se enfriaron a $4{ }^{\circ} \mathrm{C}$ durante 15 minutos, y se centrifugaron a $20 \mathrm{~g}$ durante 15 minutos. La absorbancia del sobrenadante de la mezcla y del blanco (agua desionizada) fue medida a $532 \mathrm{~nm}$ utilizando un espectrofotómetro lector de microplacas (Multiskan ${ }^{\mathrm{TM}} \mathrm{GO}$, Thermo Scientific, USA). Para realizar la curva de calibración estándar se utilizó 1,1,3,3tetraetoxipropano, precursor de MDA $\left(10^{-9}\right.$ $\mathrm{M} / \mathrm{ml})$.

\section{Análisis Estadístico}

Se trabajó con un diseño factorial y se utilizó el análisis de varianza (ANOVA) de una vía para determinar la importancia de los parámetros productivos y el ANOVA de dos vías para determinar la importancia de la prueba TBARS de los tratamientos y su interacción con los días de almacenamiento. El análisis post hoc se analizó mediante la prueba de Tukey $(\mathrm{p}<0.05)$. Los valores se expresan como la media \pm error estándar de la media. Los análisis estadísticos se realizaron utilizando el software GraphPad Prism (San Diego, CA, USA).

\section{Resultados y Discusión}

\section{Parámetros Productivos}

No se encontraron diferencias significativas entre los grupos experimentales en términos de peso del huevo (Cuadro 3). Esto es consistente con Yesilbag et al. (2013), usando aceite esencial de orégano al compararlo con otros aceites, y con los trabajos de Cetingul et al. (2009) y Christaki et al. (2012), que solo usaron niveles de polvo de orégano. Esto indica que la forma de suministro de orégano no afecta el peso del huevo en la codorniz. Esto también se observa en las gallinas ponedoras (Giannenas et al., 2005). 
El tratamiento E\&C fue el mejor en producción de huevos, seguido de C, B, A y el Control $(p<0.01)$. En el Cuadro 3 se puede observar que la mayor producción de huevos se obtienen cuanto más alto es el nivel de aceite esencial de orégano $(\mathrm{p}<0.01)$, aunque en cualquier caso superados por el grupo E\&C. Radwan et al. (2008) obtuvieron similar resultado en gallinas ponedoras al comparar varias hierbas suplementadas en polvo; sin embargo, este efecto no se evidenció en los trabajos de Yesilbag et al. (2013), Cetingul et al. (2009) y Christaki et al. (2012) en codornices, posiblemente por el uso de dosis o variedades de orégano distintas al del presente estudio debido a que se sabe cada variedad puede tener otra composición y una concentración de compuestos diferente (Toncer et al., 2009). Por otro lado, si bien el grupo $\mathrm{E} \& \mathrm{C}$ tuvo la mayor producción de huevos, He et al. (2017) obtuvo la mayor producción de huevos utilizando $150 \mathrm{mg} / \mathrm{kg}$ de aceite esencial de orégano en gallinas ponedoras, superando el resultado del grupo con antibiótico $(100 \mathrm{mg} / \mathrm{kg}$ de sulfato de colistina) en la dieta.

En masa de huevo, el grupo control tuvo el resultado más bajo $(\mathrm{p}<0.01)$, aunque Yesilbag et al. (2013) no encontraron estas diferencias en su trabajo con codornices. En el presente estudio se encontró que la masa y la producción del huevo del huevo están directamente relacionadas, ya que el grupo control obtiene la más baja producción, resultando en una menor masa de huevo (Bohren, 1970).

El grupo E\&C, juntamente con los grupos $\mathrm{C}$ y B, obtuvieron las mejores conversiones alimenticias, seguido del grupo A y del control $(\mathrm{p}<0.01)$. En el estudio de He et al. (2017), el aceite esencial de orégano fue más efectivo que el tratamiento con antibióticos. La diferencia podría ser explicada por el hecho que en este estudio se utiliza la combinación de dos antibióticos que permite una mejor efectividad de la acción antibacteriana. Por el contrario, Cetingul et al. (2009) no obtuvieron diferencias significativas entre sus tratamientos con codornices.
Con relación a la ingesta de alimento, el grupo Calcanzó el mayor consumo $(\mathrm{p}<0.01)$, posiblemente debido a una mayor palatabilidad del alimento, dada las características aromáticas del orégano (Windisch et al., 2008). Así mismo, Yesilbag et al. (2013), quienes utilizaron aceite esencial de orégano comparándolo con otros aceites obtuvieron un mayor consumo con respecto a la dieta de control. Sin embargo, otros trabajos con codornices (Cetingul et al., 2009; Christaki et al., 2012) y en gallinas ponedoras (Giannenas et al., 2005; Radwan et al., 2008; He et al., 2017) no encontraron un mayor consumo de alimento por efecto del orégano, debiendo realizarse mayores estudios para verificar este efecto.

\section{Sustancias Reactivas al Ácido Tiobar- bitúrico}

En la prueba del TBARS se encontraron diferencias altamente significativas entre los días de almacenamiento $(\mathrm{p}<0.001)$ y entre grupos $(\mathrm{p}<0.01)$, pero no en la interacción día-tratamiento (Figura 1). En un estudio similar, Botsoglou et al. (1997) reportan que la suplementación con tomillo al 3\% en la dieta de gallinas ponedoras redujo significativamente los niveles de MDA en la yema, pero sin verse influenciado por el tiempo de almacenamiento.

El grupo control presentó los niveles de MDA más altos durante todo el tiempo de almacenamiento evaluado (día $0=3.845 \times 10^{9}$ $\mathrm{M} / \mathrm{ml}$, día $7=5.058 \times 10^{9} \mathrm{M} / \mathrm{ml}$, día $15=$ $5.66 \times 10^{9} \mathrm{M} / \mathrm{ml}$ ) respecto a los demás grupos (Figura 1). Estos resultados concuerdan, puesto que se sabe que existe una relación inversa entre los niveles de malondialdehído (MDA), un indicador de la peroxidación de lípidos, y el nivel de antioxidantes en la dieta de los productos avícolas (Guo et al., 2001; Sahin et al., 2008). En el día 0 (Figura 2a) se muestra que los grupos que recibieron tratamiento con aceite esencial de orégano logran una mayor reducción $(100 \mathrm{mg} / \mathrm{kg}$ : $\mathrm{p}<0.0001$; 150 y $200 \mathrm{mg} / \mathrm{kg}$ : $\mathrm{p}<0.001)$ de los niveles de MDA que el grupo E\&C ( $<0.01)$. Resulta- 


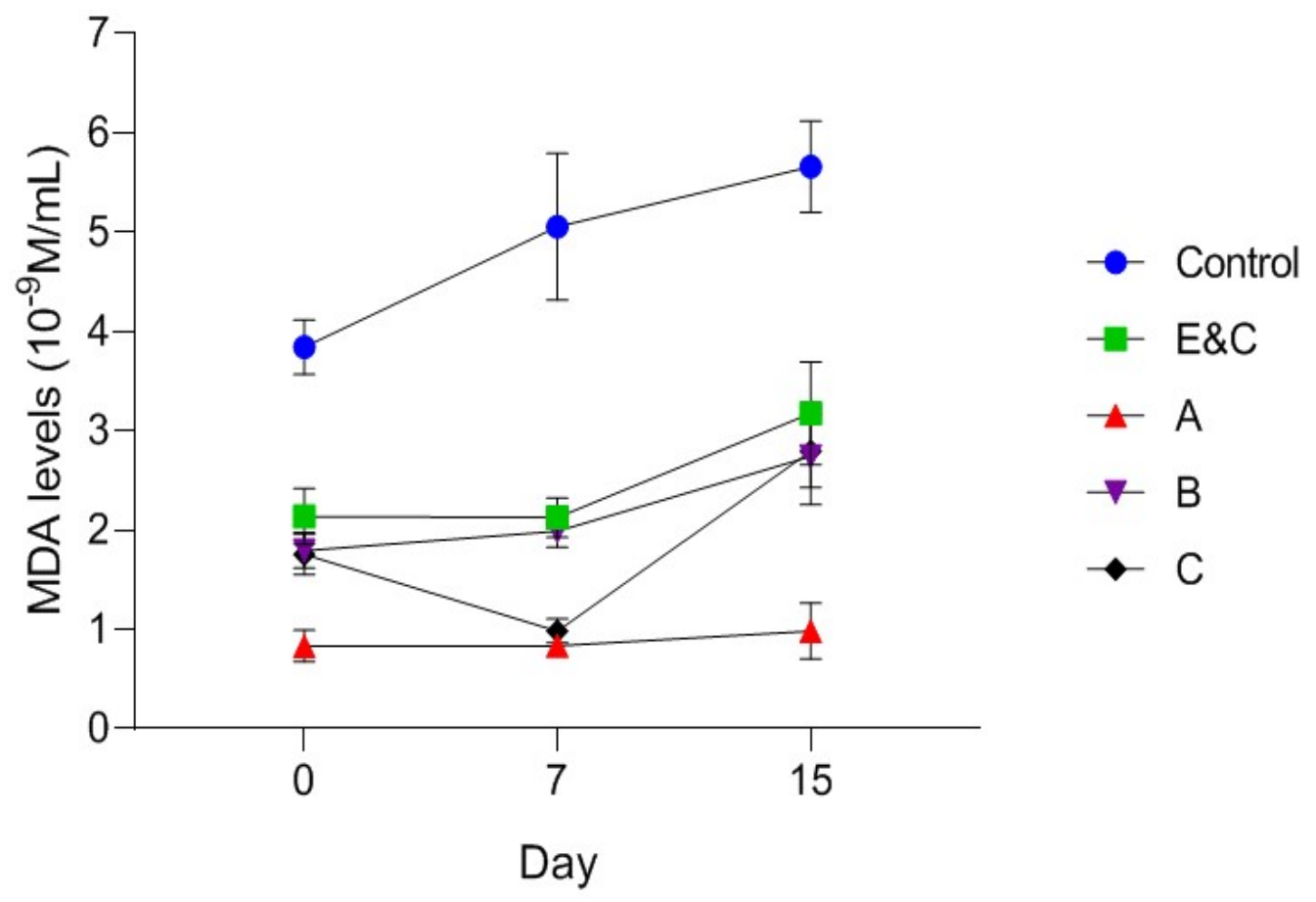

Figura 1. Influencia del tiempo de almacenamiento del huevo de Coturnix japonica en los niveles de malondialdehído (Días: $\mathrm{p}<0.0001$ ); tratamientos: $\mathrm{p}<0.0001$; interacción díatratamiento: $\mathrm{p}>0.05$ )

do similar se obtiene en los otros días de almacenamiento con excepción del día 7, donde los niveles de MDA fueron similares entre el grupo $\mathrm{C}$ y el $\mathrm{E} \& \mathrm{C}(\mathrm{p}<0.05)$. Se sabe que el aceite esencial de orégano presenta poderosos componentes antioxidantes como el timol del cual se ha reportado su posible influencia benéfica sobre el metabolismo de los lípidos y que está relacionada con la calidad nutricional de los productos como el huevo (Fernandez et al., 2019).

Respecto al grupo E\&C que recibieron promotores de crecimiento, se sabe que la colistina ha sido relacionada con neurotoxicidad, inducción de apoptosis y aumento de estrés oxidativo (Dai et al., 2017; Ajiboye et al., 2018), efectos que involucrarían a un mecanismo mediado por una disfunción mitocondrial y la regulación al alza en la expresión de los ARNm de caspasa-3 y Bax (Dai et al., 2019); razón probable por la cual los niveles de MDA son altos. En contraste, los resultados del grupo B muestran que la suplementación es más beneficiosa sobre la estabilidad oxidativa de huevo si se compara con E\&C (Figura 2a, 2b, 2c). En este sentido, Botsoglou et al. (2002), usando estos niveles de aceite de orégano, disminuyeron los niveles de MDA inducidos por hierro en muestras de tejido de pollos. Asimismo, Giannenas et al. (2005), usando la dosis de $100 \mathrm{mg} / \mathrm{kg}$, encontraron valores de MDA relativamente bajos respecto al grupo control.

Se había sugerido que el efecto antioxidante del aceite esencial de orégano sobre la peroxidación de lípidos era dependiente de la dosis, hipótesis que no fue corro- 
(a)

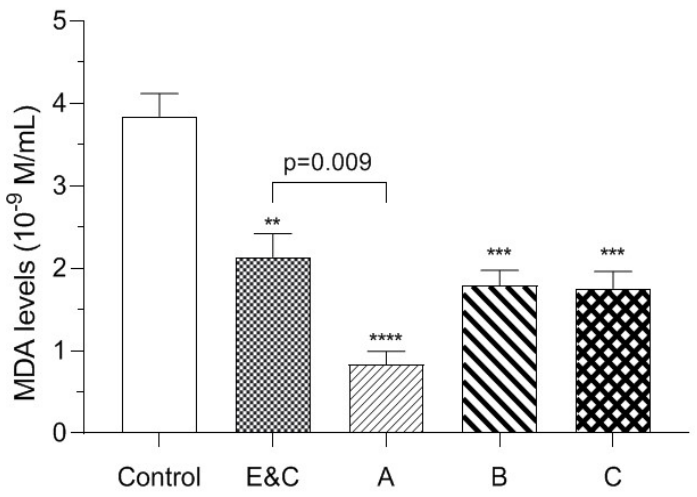

(b)

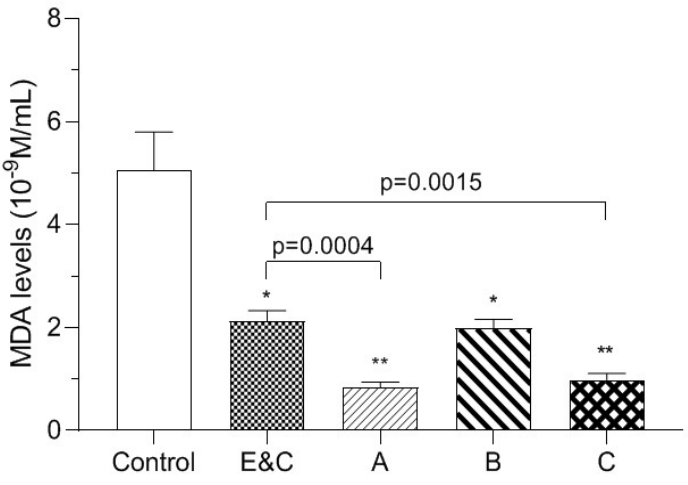

(c)

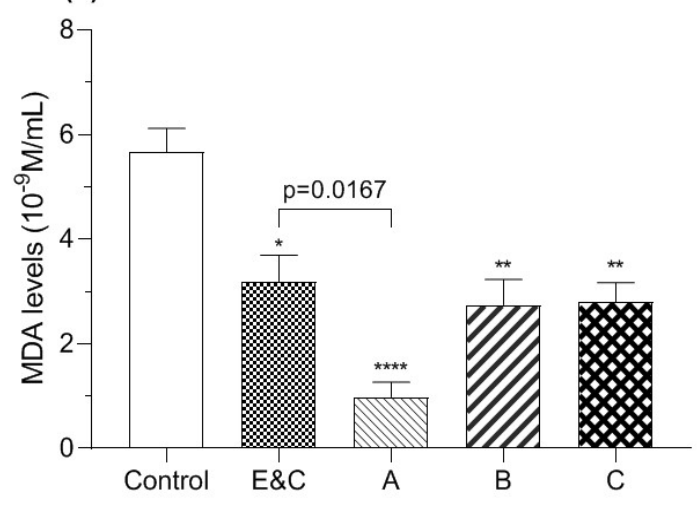

Figura 2. Niveles de malondialdehído en yema de huevo de codornices (Coturnix japónica) por día de almacenamiento. (a) día 0 ; (b) día 7; (c) día 15. (* $\mathrm{p}<0.05$, ** $\mathrm{p}<0.01, * * *$ $\mathrm{p}<0.001, * * * * \mathrm{p}<0.0001$ vs. Control)

borada en el presente estudio. Por el contrario, la dosis más baja fue la que mejor inhibió la peroxidación de lípidos en yema de huevos de codorniz, apoyando la hipótesis de que este aceite puede actuar como un bioantioxidante eficiente, incluso a dosis muy bajas como 300 ng/día (Misharina et al., 2013).

Lee et al. (2004) señalan que la posibilidad de acumulación de aceite esencial en el cuerpo es reducida debido a su rápida conversión y excreción metabólica, siempre y cuando la suplementación sea por cortos periodos. Esto se ha confirmado en productos animales como los huevos (Zhai et al., 2018).
Sin embargo, esta susceptibilidad a la oxidación de lípidos también dependería de la especie animal (Luna et al., 2010), sin dejar la posibilidad de que sea la misma en el caso de productos como el huevo de codorniz. Los resultados indicados indican una tasa moderada de oxidación de lípidos y un efecto de este tipo basado en los componentes antioxidantes del aceite esencial de orégano como el ácido rosmarínico, ácido gálico, ácido cafeico, ácido protocatecúcico, flavo-noides, timol y carvacrol, responsables de la inhibición de la oxidación de los lípidos (Yanishlieva et al., 2006). 


\section{Conclusiones}

- La suplementación con aceite esencial de orégano mejora los parámetros productivos de la codorniz respecto a la dieta control, con excepción del peso del huevo y el consumo de alimento.

- La dosis de $100 \mathrm{mg} / \mathrm{kg}$ de aceite esencial de orégano redujo significativamente la peroxidación lipídica de la yema de huevo, pero esto no fue influenciado por el tiempo de almacenamiento.

\section{Agradecimientos}

Se agradece a los propietarios de la granja de codornices y a su personal por permitir realizar esta investigación, y al $\mathrm{PhD}$ Tumen Wuliji por su contribución en la redacción de este manuscrito.

\section{Literatura Citada}

1. Ajiboye TO. 2018. Colistin sulphate induced neurotoxicity: Studies on cholinergic, monoaminergic, purinergic and oxidative stress biomarkers. Biomed Pharmacother 103: 1701-1707. doi: 10.1016/j.biopha.2018.04.189

2. Anadón A. 2006. WS14 The EU ban of antibiotics as feed additives (2006): alternatives and consumer safety. J Vet Pharmacol Ther 29: 41-44. doi: 10.1111/ j.1365-2885.2006.00775 2.x

3. Ayala A, Muñoz M, Argüelles S. 2014. Lipid peroxidation: production, metabolism, and signaling mechanisms of malondialdehyde and 4-hydroxy-2nonenal. Oxid Med Cell Longev 2014: 360438. doi: 10.1155/2014/360438

4. Beski SSM, Swick RA, Iji PA. 2015. Specialized protein products in broiler chicken nutrition: A review. Anim Nutr 1: 47-53. doi: 10.1016/j.aninu.2015.-05.005

5. Bohren BB. 1970. Genetic gains in annual egg production from selection on early part-records. Worlds Poult Sci J 26: 647-657. doi: 10.1079/wps 19700021
6. Botsoglou NA, Florou-Paneri P, Christaki E, Fletouris DJ, Spais AB. 2002. Effect of dietary oregano essential oil on performance of chickens and on iron-induced lipid oxidation of breast, thigh and abdominal fat tissues. Br Poult Sci 43: 223-230. doi: 10.1080/00071660120-121436

7. Botsoglou NA, Yannakopoulos AL, Fletouris DJ, Tserveni-Goussi AS, Fortomaris PD. 1997. Effect of dietary thyme on the oxidative stability of egg yolk. J Agric Food Chem 45: 3711-3716. doi: 10.1021/jf9703009

8. Cetingul IS, Bayram I, Yardimci M, Sahin EH, Sengor E, Akkaya AB, Uyalar C. 2009. Effects of oregano (Oregano onites) on performance, hatchability and egg quality parameters of laying quails (Coturnix coturnix japonica). Ital J Anim Sci 8: 467-477. doi: 10.4081/ijas.2009.467

9. Christaki E, Bonos E, Giannenas I, Florou-Paneri P. 2012. Evaluation of oregano and á-tocopheryl acetate on laying Japanese quail diets. J Basic Appl Sci 8: 238-242. doi: 10.6000/19275129.2012.08.01.36

10. Dai C, Tang S, Biao X, Xiao X, Chen C, Li J. 2019. Colistin induced peripheral neurotoxicity involves mitochondrial dysfunction and oxidative stress in mice. Mol Biol Rep 46: 1963-1972. doi: 10.1007/s11033-019-04646-5

11. Dai C, Ciccotosto GD, Cappai R, Wang Y, Tang S, Xiao X, Velkov T. 2017. Minocycline attenuates colistininduced neurotoxicity via suppression of apoptosis, mitochondrial dysfunction and oxidative stress. J Antimicrob Chemother 72: 1635-1645. doi: 10.1093/jac/dkx037

12. Del Rio D, Stewart A, Pellegrini N. 2005. A review of recent studies on malondialdehyde as toxic molecule and biological marker of oxidative stress. Nutr Metab Cardiovasc Dis 15:316-328. doi: 10.1016/j.numecd.2005.05.003 
13. Dibner JJ, Richards JD. 2005. Antibiotic growth promoters in agriculture: history and mode of action. Poult Sci 84: 634-643. doi:10.1093/ps/84.4.634

14. Fernandez ME, Kembro JM, Ballesteros ML, Caliva JM, Marin RH, Labaque MC. 2019. Dynamics of thymol dietary supplementation in quail (Coturnix japonica): Dataset on thymol bioavailability, egg yolk fatty acids profile and performance traits. Data Brief 24: 103884. doi: 10.1016/j.dib.2019.103884

15. Giannenas I, Koidis A, Botsoglou E, Dotas V, Mitsopoulos I, Florou-Paneri P, Nikolakakis I. 2005. Hen performance and egg quality as affected by dietary oregano essential oil and alphatocopheryl acetate supplementation. Int J Poult Sci 4: 449-454. doi: 10.3923/ ijps.2005.449.454

16. Gîrd CE, Dupu LE, Costea T, Nencu I, Popescu ML, Tudorel OO. 2016. Preliminary research concerning the obtaining of herbal extracts with potential neuroprotective activity note. I. Obtaining and characterization of a selective Origanum vulgare L. dry extract. Farmacia 64: 680-687.

17. Gonceariuc M, Balmu' Z, Benea A, Barsan V, Sandu T. 2015. Biochemical diversity of the Origanum vulgare ssp vulgare L. and Origanum vulgare ssp hirtum (link) letswaart genotypes from Moldova. JASM Life Sci 2: 92-100.

18. Guo Y, Tang Q, Yuan J, Jiang $Z$. 2001. Effects of supplementation with vitamin $\mathrm{E}$ on the performance and the tissue peroxidation of broiler chicks and the stability of thigh meat against oxidative deterioration. Anim Feed Sci Technol 89: 165-173. doi: 10.1016/S03778401(00)00228-5

19. Khan A, Bashir S, Khan SR, Gilani AH. 2011. Antiurolithic activity of Origanum vulgare is mediated through multiple pathways. BMC Complement Altern Med 11: 96. doi: 10.1186/14726882-11-96
20. He X, Hao D, Liu C, Zhang X, Xu D, $X u X$, Wang $J, W u R$. 2017. Effect of supplemental oregano essential oils in diets on production performance and relatively intestinal parameters of laying hens. Am J Mol Biol 7: 73-85. doi: 10.4236/ajmb.2017.71006

21. Lee KW, Everts H, Beynen AC. 2004. Essential oils in broiler nutrition. Int $\mathrm{J}$ Poult Sci 3: 738-752. doi: 10.3923/ ijps.2004.738.752

22. Leyva-López N, Gutiérrez-Grijalva EP, Vazquez-Olivo G, Heredia JB. 2017. Essential oils of oregano: biological activity beyond their antimicrobial properties. Molecules 22: 989. doi: 10.3390/molecules22060989

23. Lukas B, Schmiderer C, Novak J. 2015. Essential oil diversity of European Origanum vulgare L (Lamiaceae). Phytochemistry 119: 32-40. doi: 10.1016/ j.phytochem.2015.09.008

24. Luna A, Labaque MC, Zygadlo JA, Marin RH. 2010. Effects of thymol and carvacrol feed supplementation on lipid oxidation in broiler meat. Poult Sci 89: 366-370.

25. Marwat SK, Khan MA, Rehman FU, Bhatti IU. 2009. Aromatic plant species mentioned in the Holy Qura'n and Ahadith and their ethnomedicinal importance. Pak J Nutr 8: 1472-1479. doi: 10.3923/pjn.2009.1472.1479

26. Mehdi Y, Létourneau-Montminy MP, Gaucher ML, Chorfi Y, Gayatri S, Rouissi T, Brar SK, et al. 2018. Use of antibiotics in broiler production: global impacts and alternatives. Anim Nutr 4: 170-178. doi: 10.1016/j.aninu.2018.03.002

27. Millet S, Maertens L. 2011. The European ban on antibiotic growth promoters in animal feed: From challenges to opportunities. Vet J 187: 143-144. doi: 10.1016/j.tvj1.2010.05.001

28. Misharina TA, Fatkullina LD, Alinkina ES, Kozachenko AI, Nagler LG, Medvedeva IB, Goloshchapov AN, et al. 2013. Effects of low doses of essential oils on the antioxidant status of 
the erythrocytes, liver and the brain of mice. Appl Biochem Micro 50: 88-93. doi: 10.1134/s0003683814010098

29. Oniga I, Pu'ca' C, Silaghi-Dumitrescu $R$, Olah NK, Sevastre B, Marica $R$, Marcus I, et al. 2018. Origanum vulgare ssp vulgare: chemical composition and biological studies. Molecules 23: 2077. doi: 10.3390/molecules23082077

30. Papastergiadis A, Mubiru E, Van Langenhove $H$, De Meulenaer $B$. 2012. Malondialdehyde measurement in oxidized foods: evaluation of the spectrophotometric thiobarbituric acid reactive substances (TBARS) test in various foods. J Agric Food Chem 60: 9589-9594. Doi: $10.1021 / \mathrm{jf302451 \textrm {c }}$

31. Prabhakar PV, Reddy UA, Singh SP, Balasubramanyam A, Rahman MF, Kumari SI, Agawane SB, et al. 2012. Oxidative stress induced by aluminum oxide nanomaterials after acute oral treatment in Wistar rats. J Appl Toxicol 32: 436-445. doi: 10.1002/jat.1775

32. Radwan NL, Hassan RA, Qota EM, Fayek HM, 2008. Effect of natural antioxidant on oxidative stability of eggs and productive and reproductive performance of laying hens. Int J Poult Sci 7: 134-150. doi: 10.3923/ijps.2008.134.150

33. Rostagno HL, Teixeira LF, Hannas MI, Donzele JL, Zakomura NK, Perazzo FG, Saraiva A, et al. 2017. Tablas brasileñas para aves y cerdos: composición de alimentos y requerimientos nutricionales. $4^{\circ}$ ed. Brasil: Universidad Federal de Viçosa. p 347-362.

34. Sahin N, Akdemir F, Orhan C, Kucuk O, Hayirli A, Sahin K. 2008. Lycopene-enriched quail egg as functional food for humans. Food Res Int 41:295-300. doi: 10.1016/j.foodres.2007.12.006

35. Saleh AA, Ebeid TA, Abudabos AM. 2018. Effect of dietary phytogenics (herbal mixture) supplementation on growth performance, nutrient utilization, antioxidative properties, and immune response in broilers. Environ Sci Pollut Res Int 25: 14606-14613. doi: 10.1007/ s11356-018-1685-z

36. Tolik, D, Poawska E, Charuta A, Nowaczewski S, Cooper R. 2014. Characteristics of egg parts, chemical composition and nutritive value of Japanese quail eggs - A review. Folia Biol 62: 287-292. doi: 10.3409/fb62_4.287

37. Toncer O, Karaman S, Kizil S, Diraz E. 2009. Changes in essential oil composition of oregano (Origanum onites L) due to diurnal variations at different development stages. Not Bot Horti Agrobot Cluj Napoca 37: 177-181. doi: 10.15835/nbha3723188

38. Windisch W, Schedle K, Plitzner C, Kroismayr A. 2008. Use of phytogenic products as feed additives for swine and poultry. J Anim Sci 86: E140-E148. doi: 10.2527/jas.2007-0459

39. Yanishlieva NV, Marinova E, Pokorny J. 2006. Natural antioxidants from herbs and spices. Eur J Lipid Sci Technol 108: 776-793. doi: 10.1002/ej1t.200600127

40. Yesilbag D, Gezen SS, Biricik H, Meral Y. 2013. Effects of dietary rosemary and oregano volatile oil mixture on quail performance, egg traits and egg oxidative stability. Br Poult Sci 54: 231237. doi: 10.1080/00071668.2013.778389

41. Zhai H, Liu H, Wang S, Wu J, Kluenter AM. 2018. Potential of essential oils for poultry and pigs. Anim Nutr 4: 179-186. doi: 10.1016/j.aninu.2018.01.005

42. Zhang XL, Guo YS, Wang CH, Li GQ, Xu JJ, Chung HY, Ye WC, Li YL, Wang GC. 2014. Phenolic compounds from Origanum vulgare and their antioxidant and antiviral activities. Food Chem 152: 300-306. doi: 10.1016/ j.foodchem.2013.11.153 